\title{
Plantas útiles del páramo y su potencial en la sostenibilidad ambiental: caracterización etnobotánica, Sumapaz Colombia.
}

Useful plants of the paramo and their potential for environmental sustainability: ethnobotanical characterization Sumapaz Colombia.

Plantas úteis do páramo e seu potencial na sustentabilidade ambiental: caracterização etnobotánica Sumapaz Colombia.

Uriel Rodríguez Espinosa/Universidad Nacional Abierta y a Distancia, Colombia/uriel.rodriguez@unad.edu.co John Carlos Ruíz Caicedo/Universidad Nacional Abierta y a Distancia, Colombia/john.ruiz@unad.edu.co Denisse Viviana Cortés Castillo/Universidad Nacional Abierta y a Distancia, Colombia/denisse.cortes@unad.edu.co Hugo Caballero Díaz/Universidad Nacional Abierta y a Distancia, Colombia/hcaballerod@unadvirtual.edu.co

Recibido: 1/9/2020 Aceptado: 7/12/2020 Publicado: 30/12/2020

\section{RESUMEN}

En Colombia el aprovechamiento insostenible de la biodiversidad se debe al desconocimiento que existe sobre su potencial estratégico. El estudio etnobotánico que se presenta, desarrollado en la cuenca alta de la Quebrada Honda, tuvo como objetivo caracterizar el manejo de la flora local por parte de la comunidad y proponer alternativas de producción más sostenibles mediante sistemas agroforestales. Para ello, se realizaron entrevistas semiestructuradas y caminatas etnobotánicas, estas últimas con conocedores locales. Se encontraron 98 especies útiles, correspondientes a 47 familias y 86 géneros. La familia Asteraceae presentó el mayor número de especies con 14. Los géneros con mayor diversidad fueron Passiflora y Rubus. Del total de las especies identificadas, 51 tienen un uso agropecuario, 29 un uso medicinal y 28 utilizadas en servicios ambientales. Los resultados permitieron un acercamiento al conocimiento etnobotánico en zonas de amortiguación del páramo de Sumapaz, así como reconocer el conocimiento rural asociado a la biodiversidad y el agroecosistema local. Se reafirma la necesidad de incrementar el conocimiento de la comunidad sobre el potencial estratégico de las especies locales mediante la implementación de sistemas agroforestales que combinen las especies nativas con cultivos y producción pecuaria, en los que se equilibren la conservación y la producción. 
Palabras clave: conocimientos tradicionales, conservación de la flora, sistemas agroforestales, usos potenciales

\section{ABSTRACT}

The unsustainable use of Colombia's biodiversity is due to the lack of knowledge about it strategic potential. An ethnobotanical study in the high basin of Quebrada Honda river was developed, in order to characterize the uses of the flora species in the area by the community and to propose a more sustainable production alternatives through agroforestry systems. To collect the information, semi-structured interviews were conducted and ethnobotanical walks were made with local experts. Ninety eight useful species corresponding to 47 families and 86 genders were reported. The Asteraceae family presented the highest number of species with 14. Passiflora and Rubus were the highest population regarding genders. From all species studies, 51 had agricultural use, 29 medicinal use and 28 environmental services. The results obtained allowed to have an approach to the ethnobotanical knowledge in the buffer zone of the Sumapaz paramo and to recognize the rural knowledge associated to the local agroecosystem biodiversity. We empowered on the community knowledge increase regarding the strategic potential of local species trough the implementation of agroforestry systems that combine native species with a balance between conservation and production.

Keywords: agroforestry systems, flora conservation, potential uses, traditional knowledge

\section{RESUMO}

Na Colômbia, o uso insustentável da biodiversidade se deve à falta de conhecimento que existe sobre seu potencial estratégico. O estudo etnobotânico apresentado, desenvolvido na bacia alta da Quebrada Honda, objetivou caracterizar o manejo da flora local pela comunidade e propor alternativas de produção mais sustentáveis por meio de sistemas agroflorestais. Para isso, foram realizadas entrevistas semiestruturadas e passeios etnobotânicos, estes últimos com especialistas locais. Foram encontradas 98 espécies úteis, correspondendo a 47 famílias e 86 gêneros. A família Asteraceae apresentou o maior número de espécies com 14. Os gêneros com maior diversidade foram Passiflora e Rubus. Do total das espécies identificadas, 51 têm uso agrícola, 29 uso medicinal e 28 são utilizadas em serviços ambientais. Os resultados permitiram uma aproximação ao conhecimento etnobotânico em zonas de amortecimento do páramo de Sumapaz, bem como reconhecer o conhecimento rural associado à biodiversidade e ao agroecossistema local. A necessidade de aumentar o conhecimento da comunidade sobre o potencial estratégico das espécies locais é reafirmada por meio da implantação de sistemas agroflorestais que combinem as espécies nativas com a lavoura e a pecuária, nos quais se equilibra conservação e produção.

Palavras chave: conhecimento tradicional, conservação da flora, sistemas agroflorestais, usos potenciais 


\section{INTRODUCCIÓN}

Al poseer un elevado índice de biodiversidad, Colombia comparte la categoría de país megadiverso (Bello et al., 2014) junto a Argentina, Bolivia, Brasil, China, Costa Rica, Ecuador, India, Indonesia, Kenia, México, Perú, Sudáfrica y Venezuela (Andrade-C, 2011). A la vez, presenta un alto número de especies amenazadas: de las 35476 especies de fauna, 395 poseen un alto riesgo de desaparecer, al igual que 1178 de las 27881 especies de plantas. Se entiende por amenaza, la situación de sobrevivencia desfavorable para una especie o ecosistema (Kapelle, 2004). De acuerdo con Andrade-C (2011), cuando se pierde algún elemento de la biodiversidad, los ecosistemas pierden la capacidad de recuperación y los servicios que prestan se ven vulnerables.

En Colombia, la pérdida de la biodiversidad se relaciona con los cambios en el uso del territorio, la introducción y trasplante de especies, el cambio climático, disminución, pérdida o degradación de elementos de los ecosistemas nativos y agroecosistemas. Un aspecto frecuentemente subestimado respecto a este fenómeno es el uso inadecuado y la extracción de productos derivados en forma de fibras, leña, plantas medicinales, frutos y plantas ornamentales sin seguir criterios ecológicos, por citar solo algunos ejemplos.

Se suman a esta lista causal, el desconocimiento del potencial estratégico de la biodiversidad o su aprovechamiento insostenible (Andrade-C, 2011; Bello et al., 2014; Ministerio de Ambiente y Desarrollo Sostenible, 2011); lo que provoca que los ecosistemas estratégicos, como los páramos, se vean afectados en su gran mayoría por estas problemáticas, aun cuando se sabe que son ecosistemas frágiles y de alta importancia al acumular y regular el flujo del agua que suple las necesidades del ser humano y de la naturaleza (Guhl et al., 1998).

La cuenca alta de la Quebrada Honda, que abastece el recurso hídrico para 1720 hogares rurales de los municipios de Soacha, Sibaté y Granada por medio del acueducto Aguasiso E.S.P., muestra una preocupante deforestación y la pérdida del bosque primario a causa de la expansión agropecuaria (cultivos de papa, fresa y ganadería de leche), la cual ha conducido al territorio a unas condiciones de inviabilidad ambiental (Consejo Municipal de Sibaté, 2016). Ello ha generado amenazas para las especies nativas presentes en la zona, pues la comunidad hace uso de esas plantas, pero desconoce otros usos potenciales que podrían llevar a armonizar la conservación y el aprovechamiento de la flora local.

Sobre esta problemática se han realizado estudios desde la etnobotánica en las zonas de páramo de Colombia, tales como los desarrollados por Pérez y Matiz-Guerra (2017), en comunidades campesinas de Bogotá, y los de Rodríguez et al. (2018), llevado a cabo en la Sierra Nevada del Cocuy; ambos con la intención de registrar el conocimiento tradicional relacionado con la vegetación de esas áreas.

De acuerdo con lo anterior, este proyecto consiste en un estudio etnobotánico desarrollado en la cuenca alta de la Quebrada 
Honda, con el objetivo de caracterizar el manejo de la flora local por parte de su comunidad y proponer alternativas de producción más sostenibles mediante sistemas agroforestales.

\section{MATERIALES Y MÉTODOS}

\section{Área de estudio}

El estudio se desarrolló en la vereda Romeral, que forma parte de la cuenca alta de la Quebrada Honda, en jurisdicción del municipio Sibaté, Cundinamarca. Esta zona se ubica en la vertiente occidental de la cordillera Oriental a una altitud cercana a los 3000 msnm. Este sector se encuentra dentro del radio de influencia del páramo de Sumapaz y presenta en sus inmediaciones vegetación de tipo subpáramo y páramo propiamente dicho.

Figura 1. Ubicación del área de estudio.
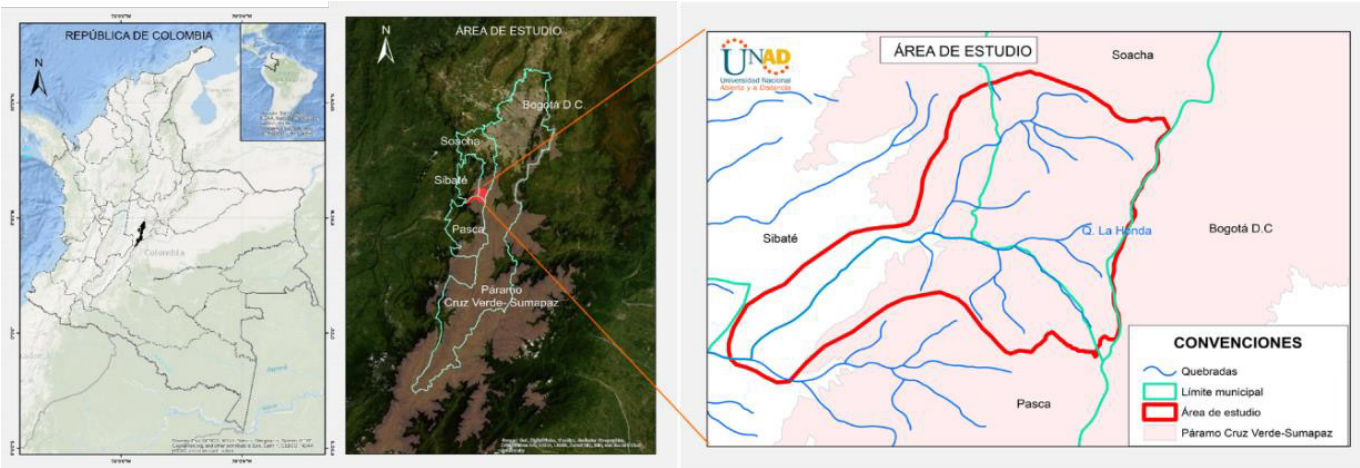

Fuente: Elaboración propia (2020).

\section{Enfoque metodológico}

Para desarrollar la investigación se siguió el enfoque metodológico de la etnobotánica, debido a la importancia científica que ha adquirido por su interdisciplinariedad $y$ aplicabilidad, especialmente en el desarrollo de procesos investigativos y productivos en los campos del conocimiento, uso, manejo y conservación de los recursos vegetales (Witte et al., 2011).

El estudio fue de carácter descriptivo teniendo en cuenta el objetivo que buscaba. En cuanto a la naturaleza de la información a recolectar, se trató de una investigación mixta que aplicó instrumentos cualitativos -entrevista semiestructurada, entrevista con lista de control y registro de relatos- e instrumentos cuantitativos -inventarios de especies vegetales en campo- (Hernández et al., 2017).

\section{Fase de campo}

Con el fin de recopilar la información sobre las formas de uso, apropiación de los recursos e iniciativas de manejo de la flora local por parte de la comunidad, así como el registro de nombres populares en el sector de estudio; se realizaron entrevistas semiestructuradas a un total de 20 familias, las cuales fueron seleccionadas bajo el criterio de vivir sobre la ronda de la Quebrada Honda y que se corresponde con el $100 \%$ de los habitantes de la misma. Además, se estableció contacto con dos sabedores locales, los cuales fueron reconocidos y recomendados por la misma 
comunidad. La fase de campo para el trabajo etnobotánico se desarrolló en 12 recorridos entre octubre de 2019 y febrero de 2020.

Dicha labor se complementó con caminatas etnobotánicas en compañía de los conocedores locales, en las cuales se recolectó el material vegetal y se anotó la información mencionada por ellos sobre el uso de las plantas. Dicha información se registró mediante grabadora y en la libreta de campo. El material botánico recolectado se cruzó con la información proveniente de los levantamientos de vegetación desarrollados en la zona. La validez taxonómica de las especies identificadas se revisó en línea a través de la herramienta Taxonomic Name Resolution Service (Iplant Collaborative, 2020).

\section{Análisis de la información}

El análisis de la información se hizo categorizando las plantas de acuerdo a los usos e importancia relativa que reportaron los entrevistados. Para los usos se siguió la metodología propuesta por Estupiñán y Jiménez (2010), la cual permite clasificar el empleo de las plantas de acuerdo con las siguientes categorías: construcción, medicinal, comestible, tecnológica, leña, servicios ambientales, agropecuario, artesanal, cerca viva, ornamental y lúdico. Para identificar la importancia relativa se siguió la metodología propuesta por Aranguren (2005), la cual permite identificar qué tan importantes son las especies para los entrevistados, de acuerdo con los siguientes criterios de calificación: muy importante, cuando una especie fue mencionada por más de 10 personas $(50 \%$ de las entrevistadas); importante, cuando la planta fue mencionada por entre tres y nueve personas diferentes; y la categoría rara, para las especies que fueron mencionadas por dos o menos personas.

\section{RESULTADOS Y DISCUSIÓN}

\section{Caracterización florística}

Se reportaron 98 especies útiles para la comunidad de la cuenca alta de la Quebrada Honda. Estas especies corresponden a 47 familias y 86 géneros. La familia Asteraceae presentó el mayor número de especies con 14, seguida por Poaceae con ocho, Melastomataceae y Rosaceae con cinco, y Lamiaceae con cuatro. Los géneros con mayor número de especies fueron Passiflora y Rubus con tres cada una. Otros géneros como Baccharis, Diplostephium, Escallonia, Hypericum, Mentha, Miconia, Morella y Rumex presentaron dos especies respectivamente. Los demás géneros estuvieron representados por una sola.

Estos datos coinciden con los estudios realizados por Galvis y Torres (2017) en comunidades rurales de Sogamoso; y de Rodríguez et al. (2018) en el ecosistema de páramo en la Sierra Nevada del Cocuy, donde la Asteraceae es la familia más importante, debido al uso medicinal que se da por parte de la comunidad, lo cual demuestra la relevancia de esta familia para las comunidades rurales que habitan las zonas de amortiguación del páramo. Igualmente, en cuanto a la representatividad por género, se destacan Diplostephium, Hypericum y Miconia, situación que coincide con el patrón de riqueza de este ecosistema 
en Colombia, descrito por Rangel (2018).

Se analizó el origen de las especies reportadas y se encontró que el $57 \%$ de las utilizadas por los habitantes del área son nativas, el $20 \%$ cultivadas y el $14 \%$ naturalizadas. También se evidenció que el $5 \%$ de las especies se consideran endémicas en el país; dichas especies son: Hesperomeles goudotiana (Decne), Killip, Ageratina aristeii (B.L. Rob.) R.M. King \& H. Rob., Greigia stenolepis L.B. Sm. y Blakea quadrangularis Triana. El porcentaje de especies nativas reportadas en las entrevistas es inusual, dado que, de acuerdo con Rodríguez et al. (2018), la proporción de plantas nativas usadas es inferior al de las especies introducidas; este estudio demuestra que hay un acervo de conocimiento en relación con la biodiversidad propia de la zona.

En cuanto al estado de conservación, 11 especies se encuentran en la categoría de preocupación menor (LC), una en vulnerable (VU) y una casi amenazada (NT) (Bernal et al., 2019). Su estado de conservación no se ha evaluado; sin embargo, los usos reportados para las mismas están relacionados con la protección de la cuenca, de manera que no se espera afectación sobre las poblaciones locales (tabla 1).

Tabla 1. Estado de conservación de las plantas de acuerdo con la Unión Internacional para la Conservación de la Naturaleza, UICN.

\begin{tabular}{llc}
\hline Nombre científico & Nombre común & Categoría UICN* \\
\hline Sambucus nigra L. & Sauco & LC \\
Baccharis latifolia (Ruiz \& Pav.) Pers. & Chilco paramuno & LC \\
Phytolacca bogotensis Kunth. & Guaba o Guava & LC \\
Chenopodium ambrosioides L. & Paico & LC \\
Passiflora mixta L. f. & Curubo montañero & LC \\
Quercus humboldtii L. & Roble & VU \\
Ambrosia peruviana All. & Artemisa & LC \\
Niphogeton ternata (Willd. ex Schltr.) Mathias & Apio de monte & LC \\
\& Constance. & & \\
Plantago major L. & Llantén & LC \\
Greigia stenolepis L.B. Sm. & Piñuela & NT \\
Passiflora tripartita (Juss.) Poir. & Curuba & LC \\
Clinopodium brownei (Sw.) Kuntze & Poleo & LC \\
Clusia multiflora Kunth & Gaque & LC \\
\hline
\end{tabular}

Nota: *LC: Preocupación menor; VU: Vulnerable; NT: Casi amenazada

Fuente: Elaboración propia (2020). 


\section{Categorías de uso}

Con respecto a los usos reportados (figura 2), se encontró que el mayor de ellos está asociado a la categoría de uso agropecuario con 51 especies, seguido por medicinal con 29 y servicios ambientales con 28 especies.
La categoría de uso agropecuario es la de mayor relevancia en el estudio y esto viene dado por las actividades propias de la zona, donde las actividades económicas son las agrícolas y las pecuarias, principalmente.

Figura 2. Número de plantas por categoría de usos.

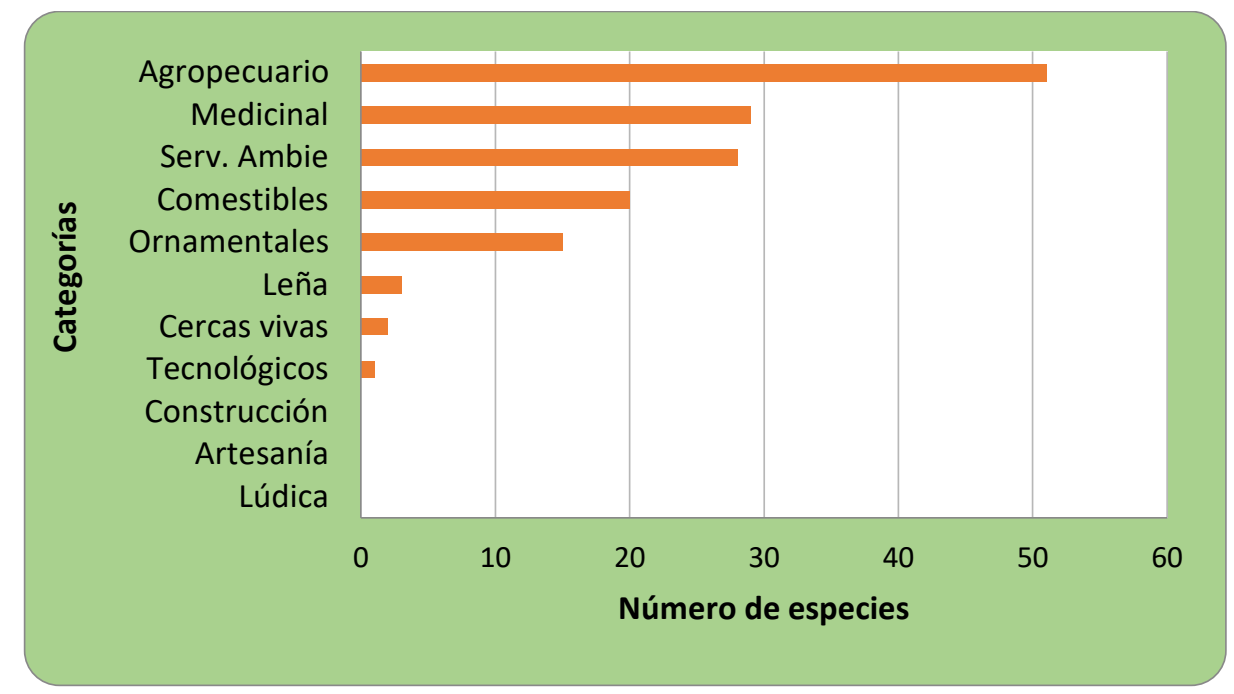

Fuente: Elaboración propia (2020).

Dentro de los usos agropecuarios se encuentran especies utilizadas para la alimentación en sistemas productivos pecuarios de mediana y pequeña escala, destacándose herbáceas como Lolium sp. L., Holcus lanatus L., Avena sativa L. y otras especies leñosas como Sambucus nigra L. y Hesperomeles goudotiana (Decne.) Killip las cuales se usan en ramoneo para bovinos. Otro uso agropecuario está relacionado con la salud animal. Los entrevistados mencionan el uso de Diplostephium rosmarinifolium (Benth.) Wedd, y Phytolacca bogotensis Kunth para aliviar afecciones en la ubre de los bovinos.

La categoría de usos medicinales es la segunda en importancia para los pobladores de la cuenca. Las plantas cultivadas en los huertos tienen ese fin para las familias. En este sentido, la Mentha piperita L., M. arvensis L. y Sonchus oleraceus L. son usadas para aliviar dolores estomacales; Borago officinalis L. para aliviar afecciones respiratorias; Seneciosp L., para sanar heridas y $\mathrm{H}$. goudotiana L. es utilizada para aliviar problemas respiratorios causados por gripe. Al comparar estos resultados con otros estudios etnobotánicos se encuentra que la categoría medicinal se reporta como la más importante, según Rodríguez et al. (2018) y Galvis y Torres (2017); sin embargo, en la presente investigación ella se ubica en segundo lugar, debido a la tradición agropecuaria de la zona.

Es importante resaltar que, del total de especies reportadas, el $28.6 \%$ se asocian a 
la categoría de uso en servicios ambientales, más específicamente en la conservación de la cuenca, lo cual es altamente positivo si se compara con estudios realizados en otros ecosistemas, tales como los de Estupiñán y Jiménez (2010), y Pérez y Matiz-Guerra (2017), donde esta categoría no sobrepasa el $14 \%$. No obstante, lo que se observa en la zona analizada es que, con la ampliación de la frontera agrícola, por medio de los arados y la preparación del suelo para el cultivo de papa (Solanum tuberosum L.), los árboles allí presentes son poco valorados y por lo tanto desaparecen quedando los suelos limpios para el cultivo, realidad que se repite en la mayoría de los páramos del país (Garavito, 2015).

Esta situación permite inferir que no necesariamente el conocimiento del uso que se da a una especie significa su conservación, lo cual tributaría a que la misma no sea expuesta a situaciones de amenaza. Las comunidades pueden conocer para qué sirve una planta, pero si la conservación de esta se enfrenta a la posibilidad de establecer un cultivo de alta rentabilidad, son pocas las posibilidades de conservarla. Dicho escenario enfrenta la producción a la conservación en una ruralidad carente de las oportunidades que permitan elevar sus ingresos económicos.

Esa realidad afianza la necesidad de establecer líneas de acción que lleven a la comunidad a dimensionar el valor estratégico de estas especies, de manera que se genere una cultura donde la necesidad no justifique las presiones y amenazas a aquellas que cumplen un papel clave en el ecosistema, y que aseguran recursos como el hídrico, tan importante para el propio desarrollo socioeconómico de la comunidad.

Con respecto a las especies que presentan mayor empleo se destacó el mortiño $(H$. goudotiana L.). Esta especie registró cinco categorías de uso: agropecuaria (ramoneo), comestible (consumo del fruto), medicinal (tratamiento de la tos), ornamental y servicios ambientales (protección de cuencas). El chilco paramuno Baccharis latifolia (Ruiz \& Pav.) Pers., y la uva camarona Macleania rupestris (Kunth) A.C. Sm., presentaron también cinco categorías de uso: agropecuaria (ramoneo), ornamental, servicios ambientales (protección de cuencas), medicinal (tratamiento de la tos) y comestible (consumo del fruto), respectivamente. Por otra parte, el sauco (Sambucus nigra L.) presentó tres categorías de uso: agropecuaria (ramoneo), cercas vivas y medicinal (tratamiento de la tos).

Por otra parte, los recorridos etnobotánicos evidenciaron que existen otros empleos de las plantas poco expresados por los entrevistados. Es el caso del uso de árboles talados como leña o para la construcción de cercas. Es evidente que, en las fincas del sector, el principal combustible para cocer los alimentos es la leña, situación que no se aleja de la realidad continental (Food and Agriculture Organization [FAO], 2008); además, se comprobó que las divisiones entre fincas, potreros y bordes de carreteras se hacen con cercas de púas soportadas en postes de madera. En ambos casos, la materia prima son árboles de la zona que se talan para estos fines.

En ambos hallazgos se estiman dos posibles razones. Por una parte, que estos usos están 
altamente arraigados a la tradición y al día a día de los habitantes de manera que ya no se dimensionan como usos claves e importantes para ellos; por la otra, que las restricciones de las autoridades ambientales han llevado a que la comunidad no exprese abiertamente el empleo de árboles para tales fines, ni siquiera frente a estudios investigativos. Sendos casos ponen de manifiesto la necesidad de aceptar estos usos, medir sus impactos y dimensionar la urgencia de encontrar alternativas que integren árboles que puedan utilizarse como leña y madera en los sistemas productivos, sin amenazar la conservación de las especies.

De acuerdo con lo anterior, en cuanto a la necesidad de encontrar estrategias que permitan la conservación de las especies que cumplen un papel clave en este ecosistema, integrándolas a los sistemas productivos de manera que se puedan aprovechar sin amenazar la estructura ecológica y la biodiversidad local, se perfilan los sistemas agroforestales como una de las alternativas más acertadas, pues como lo indica la FAO (2018), si los sistemas agroforestales se diseñan y se gestionan de manera adecuada pueden contribuir a la conservación de la biodiversidad. Además, es evidente la necesidad de transformar las formas de producción enfocadas a las propuestas en los Objetivos de Desarrollo Sostenible, donde la conservación de los recursos, y al mismo tiempo, el aumento de la producción, pueden aportar a minimizar el hambre y mejorar los medios de subsistencia (FAO, 2019).

En ese sentido, de acuerdo con las especies caracterizadas, se consideran pertinentes los sistemas agroforestales tipo silvopastoril, combinando especies frutales como $H$. goudotiana (Decne.) Killip y M. rupestris (Kunth) A.C. Sm., que pueden aportar alimento para la familia y la avifauna; y especies leñosas como S. nigra L., A. acuminata Kunth y B. Iatifolia (Ruiz \& Pav.) Pers., que pueden aportar sombra, leña y forraje. También es posible reforzar la siembra de especies medicinales en las huertas de las viviendas, como alternativa para la conservación del conocimiento, las tradiciones de la comunidad y los recursos que pueden servir para atender necesidades como las medicinales, como lo son las especies Mentha piperita L., M. arvensis L., Sonchus oleraceus L.

Sin duda se requiere utilizar las especies a fin de solventar necesidades, pero haciendo un aprovechamiento sostenible de las mismas, a lo cual puede aportar su cultivo, aun cuando esto exige una labor y esfuerzo adicional para la comunidad, diferente al obtenerlas directamente del bosque.

\section{Valor de importancia}

De acuerdo con los criterios establecidos por Aranguren (2005), la información mostró 70 especies raras (71\%), 26 importantes (26\%) y dos muy importantes. El sauco (S. nigra L.) es la especie con mayor importancia relativa con 14 menciones, seguida por Lolium sp. L. con 10 menciones. Dentro de las especies importantes se destacaron $H$. lanatus L., A. sativa L., y las especies protectoras de la cuenca como el Alnus acuminata Kunt, $B$. latifolia (Ruiz \& Pav.) Pers., H. goudotiana (Decne.) Killip y Weinmmannia tomentosa L. f., las cuales son nativas de la zona. 
Tabla 2. Especies importantes y muy importantes según número de menciones.

\begin{tabular}{lccc}
\hline Nombre científico & Nombre común & Menciones & Valor relativo \\
\hline Sambucus nigra L. & Sauco & 14 & Muy importante \\
Lolium sp. L. & Pasto Ray Grass & 10 & Muy importante \\
Holcus lanatus L. & Pasto Poa & 9 & Importante \\
Alnus acuminata Kunth & Aliso & 8 & Importante \\
Avena sativa L. & Avena & 8 & Importante \\
Baccharis latifolia (Ruiz \& Pav.) Pers. & Chilco paramuno & 7 & Importante \\
Hesperomeles goudotiana (Decne.) Killip & Mortiño & 6 & Importante \\
Weinmannia tomentosa L. f. & Encenillo & 6 & Importante \\
Diplostephium rosmarinifolium (Benth.) Wedd. & Romero & 5 & Importante \\
Eucalyptus globulus Labill. & Eucalipto & 5 & Importante \\
Calendula officinalis L. & Caléndula & 4 & Importante \\
Mentha piperita L. & Yerbabuena & 4 & Importante \\
\hline
\end{tabular}

Fuente: Elaboración propia (2020).

La S. nigra L. es la especie más nombrada por los entrevistados y gana importancia por los usos medicinales y pecuarios que posee. Si bien no es una especie nativa (Bernal et al., 2019), se ha adaptado bien a estos lugares y la comunidad la identifica con facilidad, de allí que su empleo se evidencie en gran parte de la cuenca. Los pastos como Lolium sp. L. y el H. lanatus L. son el principal alimento de los animales en las fincas, de allí la importancia que representa para la comunidad.

Las especies $A$. acuminata Kunth, B. latifolia (Ruiz \& Pav.) Pers., H. goudotiana (Decne.) Killip, y $W$. tomentosa L.f., se encuentran principalmente en las zonas de reserva, en las inmediaciones al páramo de Sumapaz, y son reconocidas por la comunidad como especies para el cuidado de la cuenca. Por su parte, el Eucalyptus globulus Labill. es importante por sus características de fácil adaptación y rápido crecimiento, pero con restricción de siembra al ser una especie introducida. Dentro del criterio de especies importantes también se encuentran algunas relacionadas al uso medicinal, propagadas en huertos alrededor de las viviendas, tales como: D. rosmarinifolium (Benth.) Wedd., M. piperita L., Calendula officinalis L.

Los resultados anteriormente presentados evidencian que la importancia de las especies para la comunidad del área de estudio está en función de los usos que pueden hacer de estas, específicamente, los usos inmediatos como medicina o alimento para los animales. Si bien se reportaron 98 especies como reconocidas, 70 de estas son raras, según criterio de importancia; lo que sugiere un desconocimiento sobre su potencial por parte de la comunidad, 
de lo que pueden significar en su entorno inmediato y en sus unidades productivas. Por otra parte, las especies identificadas como muy importantes e importantes se pueden implementar en sistemas agroforestales, con distintos arreglos, de manera que se genere un aprovechamiento sostenible y se preserve la biodiversidad de la zona.

\section{CONCLUSIONES Y RECOMENDACIONES}

Este estudio permitió un acercamiento al conocimiento etnobotánico de especies en la zona de amortiguación del páramo de Sumapaz, específicamente en la cuenca alta de la Quebrada Honda; y muestra la importancia del conocimiento rural asociado a la biodiversidad y el agroecosistema local como insumo estratégico para la conservación y aprovechamiento sostenible de las especies propias del territorio.

De manera general, las familias que participaron en el estudio dan uso a las especies vegetales de acuerdo con su actividad agropecuaria tradicional, principalmente, de acuerdo con las necesidades medicinales y alimenticias. El conocimiento de las formas de uso se evidencia más en las personas adultas que en las jóvenes, por lo que este conocimiento local debe ser tenido en cuenta en el momento de definir las estrategias que permitan continuar usando las especies nativas de una forma adecuada y sostenible.

Lo anterior reafirma la necesidad de incrementar el conocimiento que la comunidad tiene en cuanto al potencial de uso de las especies de la zona; pero no limitándolas solamente al uso, pues esto generaría mayores problemas de deforestación y amenaza para las especies, sino reorientándola hacia su conservación mediante el incremento de su cultivo y el aprovechamiento sostenible de las mismas, lo cual se puede obtener mediante la implementación de sistemas agroforestales que combinen especies nativas con los cultivos y la producción pecuaria en los que se equilibren la conservación y la producción.

\section{REFERENCIAS BIBLIOGRÁFICAS}

Andrade-C, M.G. (2011). Estado del conocimiento de la biodiversidad en Colombia y sus amenazas. Consideraciones para fortalecer la interacción ciencia-política. Revista de la Academia Colombiana de Ciencias Exactas, Físicas y Naturales, 35(137), 491-507. https://bit. Iy/3p6i3Ev

Aranguren, A. (2005). Plantas útiles empleadas por los campesinos de la región de Bailadores, Venezuela. Boletín Antropológico, 23(64), 139-165. https://bit.ly/37JW14Q

Bello, J.C., Báez, M., Gómez, M.F., Orrego, O., y Nägele, L. (Eds.). (2014). Biodiversidad 2014. Reporte de estado y tendencias de la biodiversidad continental en Colombia. Instituto Alexander von Humboldt. https://bit.ly/2K4RhOL

Bernal, R., Gradstein, S.R., y Celis, M. (Eds.). (2019). Catálogo de plantas y líquenes de Colombia. Instituto de Ciencias Naturales-Universidad Nacional de Colombia. https://bit.ly/3aGoBG7 
Consejo Municipal de Sibaté. (2016). Plan de Desarrollo Municipal 2016-2019. Departamento de Cundinamarca, Colombia. https://bit.ly/38dxPay

Estupiñán, A., y Jiménez, N. (2010). Uso de las plantas por grupos campesinos en la franja tropical del Parque Nacional Natural Paramillo (Córdoba, Colombia). Caldasia, 32(1), 21-38. https:// bit.ly/3nOoBro

Food and Agriculture Organization. (2008). Bosques y energía: cuestiones claves. https://bit. ly/3gZTruE

Food and Agriculture Organization. (2018). Conjunto de herramientas para la Gestión Forestal Sostenible. https://bit.ly/2KfOb9z

Food and Agriculture Organization. (2019). Transformar la alimentación y la agricultura para alcanzar los ODS. https://bit.ly/3rOPvy6

Galvis, M., y Torres, M. (2017). Etnobotánica y usos de las plantas de la comunidad rural de Sogamoso, Boyacá, Colombia. Revista de Investigación Agraria y Ambiental, 8(2), 187-206. https://doi.org/10.22490/21456453.2045

Garavito, L.N. (2015). Los páramos en Colombia, un ecosistema en riesgo. Ingeniare, (19), 127-136. https://doi.org/10.18041/1909-2458/ingeniare.19.530

Guhl, E., Wills, E., Macías, L.F., Boada, A., y Capera, C. (1998). Guía para la gestión ambiental regional y local. FONADE-Departamento de Planeación Nacional-Quinaxi. https://bit.ly/34nvVmd

Iplant Collaborative. (2020, 30 de agosto). Taxonomic Name Resolution Service v4.1 [en línea]. https://bit.ly/3hfwZh3

Kapelle, M. (2004). Diccionario de la Biodiversidad. Instituto Nacional de Biodiversidad y Cooperación Española. INBio Press. https://bit.ly/2WpUA55

Ministerio de Ambiente y Desarrollo Sostenible. (2011). Política nacional para la gestión integral de la Biodiversidad y sus Servicios Ecosistémicos. Instituto Alexander von Humboldt. https://bit.ly/37MigeE

Pérez, D. y Matiz-Guerra, L. (2017). Uso de las plantas por comunidades campesinas en la ruralidad de Bogotá D.C., Colombia. Caldasia, 39(1), 68-78. https://doi.org/10.15446/caldasia. v39n1.59932

Rangel, J.O. (2018). Las plantas con flores del páramo colombiano. En Colombia diversidad biótica XVI. Patrones de riqueza y de diversidad de las plantas con flores en el bioma de páramo. Universidad Nacional de Colombia. https://bit.ly/37swgFP

Rodríguez, M., Angueyra, A., Cleef, A., \& Van Andel, T. (2018). Ethnobotany of the Sierra Nevada del Cocuy-Güican: climate change and conservation strategies in the Colombian Andes. Journal of Ethnobiology and Ethnomedicine, 14, 34. https://doi.org/10.1186/s13002-018-0227-6 
Hernández, R., Mendoza, Ch.P., Méndez, S. y Cuevas, A. (2017). Fundamentos de investigación. McGraw-Hill.

Witte, S.L., Sanabria, O.L., Chacon, P., y García, R. (Eds.). (2011). Manual de Herramientas Etnobotánicas relativas a la Conservación y el Uso Sostenible de los Recursos Vegetales. Una contribución de la Red Latinoamericana de Botánica a la Implementación de la Estrategia Global para la Conservación de las Especies Vegetales hacia el logro de las Metas 13 y 15. OEA-FEMCIDI-RLB. http://www.ibiologia.unam.mx/gela/manualetnobot.pdf

\section{AGRADECIMIENTOS}

Expresamos nuestro sincero agradecimiento al acueducto Aguasiso E.S.P por el apoyo a esta investigación, así como a los habitantes y familias de la vereda Romeral, quienes nos compartieron sus conocimientos y prácticas culturales. También a la Universidad Nacional Abierta y a Distancia (UNAD), por el decidido apoyo al desarrollo de este proyecto. 